\title{
Prognostic significance of aqueous humour lactic dehydrogenase activity
}

\author{
P. L. R. DIAS \\ From the Department of Physiology, Faculty of Medicine, University of Sri Lanka, Colombo 8, Sri Lanka
}

SUMMARY The lactic dehydrogenase (LDH) activity of the aqueous humour has been estimated in both eyes of 7 patients having uniocular retinoblastoma. In 1 patient the aqueous humour LDH activity in the healthy eye was above normal, but there was no clinical evidence of malignancy. Tumour tissue was detected in this eye 9 months later, and the aqueous humour showed a rise in LDH activity. A high LDH activity persisted even after irradiation, though no tumour tissue was visible ophthalmoscopically. It is suggested that the estimation of the LDH activity in the aqueous humour of the healthy eye in cases of uniocular retinoblastoma might be of value in the early detection of a bilateral retinoblastoma.

The diagnostic significance of an increase in the lactic dehydrogenase (LDH) activity in the aqueous humour was first demonstrated by Dias et al. (1971). Since then several other workers have confirmed these findings (Kaneko and Suzuki, 1972; Swartz et al., 1974; Kabak and Romano, 1975; Felberg et al., 1977). An increased LDH activity, however, has also been reported in other nonmalignant conditions associated with intraocular cell necrosis (Stone and Krupin, 1976). There is no doubt that, together with other ancillary investigations, the estimation of LDH activity in the aqueous humour is an invaluable procedure in the diagnosis of retinoblastoma.

Since about $25 \%$ of the cases of retinoblastoma are bilateral, it was decided to investigate the LDH activity in the aqueous humour of the healthy eye in patients with established unilateral retinoblastoma, to determine whether the estimation of $\mathbf{L D H}$ activity would be of help in the early diagnosis of retinoblastoma in the healthy eye.

\section{Materials and methods}

Aqueous humour was collected by aqueous puncture from both eyes of 7 patients who presented at the Victoria Memorial Eye Hospital, Colombo, with a white mass clinically diagnosed as a retinoblastoma behind 1 pupil, the other eye being clinically completely normal. The specimens of aqueous humour were kept at $4^{\circ} \mathrm{C}$ and analysed the same day.

The LDH activity was estimated by the colorimetric method of King (1959), in which the increase in optical density at $340 \mathrm{~nm}$ was measured. A unit of activity is the amount of LDH which reduces $1 \mu$ mole lactate to $1 \mu$ mole pyruvate in 15 minutes at $37^{\circ} \mathrm{C}$. Activity is expressed in units per $100 \mathrm{ml}$ of aqueous humour (SI conversion: units $/ 1=$ units/ $100 \mathrm{ml} \times 10$ ). Care was taken to see that none of these specimens was contaminated with blood.

\section{Results}

The LDH activity of the aqueous humour in the 7 eyes containing the white mass ranged between 1460 and 3400 units $/ 100 \mathrm{ml}$, and were all considered to be in the malignant range (values over 1000 units $/ 100 \mathrm{ml}$ were regarded as being malignant [Dias et al., 1971]). These eyes were all enucleated, and subsequent histology showed a retinoblastoma in each case.

The aqueous humour removed from the healthy eyes of 6 of these 7 patients ranged between 180 and 420 units $/ 100 \mathrm{ml}$. The normal range is 160 to 400 units $/ 100 \mathrm{ml}$ (King, 1959). In 1 of these patients with unilateral retinoblastoma, however, the aqueous humour removed from the healthy eye showed an LDH activity of 840 units $/ 100 \mathrm{ml}$. This level, though above the normal range, was below the range considered to be malignant. The case history of this child is recorded below.

CASE REPORT

A girl aged $1 \frac{1}{2}$ years was brought to the Eye Hospital,

Address for reprints: Dr P. L. R. Dias, 4 Tower View, Sale Hill, Sheffield 10. 
Colombo, in May 1977 with a history of a white mass behind the left pupil of 6 weeks' duration. The pupil was dilated and fixed. The tension was increased, the anterior chamber was deep, and the iris showed atrophic patches. Floccules of necrotic material were present in the vitreous, and the retina was completely infiltrated by a yellowish white vascular mass producing a solid retinal detachment. Clinically the right eye was perfectly normal, and there was no traceable family history of retinoblastoma.

A diagnostic aqueous puncture done on the left eye revealed an LDH activity of 2360 units $/ 100 \mathrm{ml}$, confirming that the mass was most likely to be a retinoblastoma. The eye was enucleated, and at the time of enucleation aqueous humour was removed from the clinically normal right eye for LDH assay. The LDH activity of the aqueous humour in the right eye was 840 units $/ 100 \mathrm{ml}$, and histological examination of the left eye showed an undifferentiated retinoblastoma with areas of necrosis and calcification but showing no invasion of the optic nerve or choroid.

The child was discharged from hospital in July 1977, but through default was followed up very irregularly. In February 1978 it was observed that there was a small yellowish mass near the posterior pole with a well-defined temporal edge. A diagnostic aqueous puncture was performed, and the LDH activity of the aqeuous humour at this stage was 1170 units $/ 100 \mathrm{ml}$. The child was given external cobalt therapy of 3500 rads over 4 weeks to the right eye. (Cobalt plagues are not available in Sri Lanka.)

In May 1978 examination of the right eye revealed no trace of the mass at the posterior pole, and an estimation of the LDH activity in this eye showed it to be 760 units $/ 100 \mathrm{ml}$.

\section{Discussion}

The initial LDH estimation in the clinically normal right eye, which was of a value higher than normal, probably suggests the presence of a tumour which was too small to be seen ophthalmoscopically but which was actively secreting LDH. It is known that malignant cells are capable of actively secreting LDH into the surrounding body fluids (Wroblewski, 1957). The increase in the LDH activity of the aqueous humour in this eye during a period of 9 months leaves no doubt as to the occurrence of a malignant tumour in the right eye.

The reason for the subsequent persistence of the LDH activity of the aqueous humour in the right eye at a level higher than normal even 2 months after irradiation is difficult to explain. It is probably due to the lysis of cells caused by irradiation and the release of intracellular LDH, which, being globulin in nature, is only slowly removed from the aqueous humour; or it could indicate the persistence of remnants of tumour tissue too small to be seen ophthalmoscopically but still actively secreting LDH.

It is well known that the serum creatine phosphokinase (CPK) levels are very high in children who have the Duchenne type of muscular dystrophy. These enzyme levels are very high in children predisposed to dystrophy even at birth, long before any signs or symptoms of dystrophy are clinically detectable (Smith et al., 1966). Raised enzyme levels in asymptomatic children having a family history of dystrophy are therefore of diagnostic significance. Abnormal carcinoembryonic antigen levels have been demonstrated in family members of cases of retinoblastoma showing no clinical or ophthalmoscopic manifestations (Felberg et al., 1976).

Since retinoblastomas are associated with a very high LDH activity, it is likely that the LDH activity in the aqueous humour of the healthy eye would be raised in the very early stages of a case of retinoblastoma, even before the tumour mass is visible ophthalmoscopically. It is suggested that the LDH activity be routinely estimated in the healthy eye of patients having unilateral retinoblastoma even though the healthy eye may appear clinically and ophthalmoscopically normal, for this may help in the early detection of a bilateral retinoblastoma and thus greatly improve the prognosis through early treatment. There could possibly even be a place in the future for 'prophylactic radiotherapy' to the healthy eye in patients with unilateral retinoblastoma who show a raised LDH activity in the healthy eye even without any clinical or ophthalmoscopic manifestations.

I thank the surgeons at the Victoria Memorial Eye Hospital, Colombo, who extended to me their fullest co-operation, and Miss Cheryl Fernando for secretarial assistance.

\section{References}

Dias, P. L. R., Shanmuganathan, S. S., and Rajaratnam, M. (1971). Lactic dehydrogenase activity of aqueous humour in retinoblastoma. British Journal of Ophthalmology, 55, 130-132.

Felberg, N. T., Michelson, J. B., and Shields, J. A. (1976). CEA family syndrome: abnormal carcinoembryonic antigen (CEA) levels in asymptomatic retinoblastoma family members. Cancer, 37, 1397-1402.

Felberg, N. T., McFall, R., and Shields, J. A. (1977). Aqueous humour enzyme patterns in retinoblastoma. Investigative Ophthalmology, 16, 1039-1046.

Kabak, J., and Romano, P. E. (1975). Aqueous humour lactic dehydrogenase isoenzymes in retinoblastoma. British Journal of Ophthalmology, 59, 268-269.

Kaneko, A., and Suzuki, H. (1972). Lactic acid dehydrogenase activity and isoenzyme in the retinoblastoma. 
Acta Societatis Ophthalmologicae Japonicae, 76, 672-675. King, J. (1959). A routine method for the estimation of lactic dehydrogenase activity. Journal of Medical Laboratory Technology, 16, 265-272

Smith, H. L., Amick, L. D., and Johnson, W. W. (1966). Detection of subclinical and carrier states in Duchenne muscular dystrophy. Journal of Pediatrics, 69, 67-74.

Stone, R. A., and Krupin, T. (1976). Elevated lactic acid dehydrogenase in aqueous humour in an eye without retinoblastoma. American Journal of Ophthalmology, 82, 94-96.

Swartz, M., Herbst, R. W., and Goldberg, M. F. (1974). Aqueous humour lactic acid dehydrogenase in retinoblastoma. American Journal of Ophthalmology, 78, 612617.

Wroblewski, F. (1957). The clinical significance of alteration in lactic dehydrogenase activity of body fluids. American Journal of Medical Science, 234, 301-312. 